Témoigner Témoigner. Entre histoire et mémoire

Getuigen Revue pluridisciplinaire de la Fondation Auschwitz

119 | 2014

II y a 70 ans, Auschwitz. Retour sur Primo Levi

\title{
Entre témoignage et mémoire, quelle place pour Primo Levi?
}

Between testimony and memory, what place for Primo Levi?

Tussen getuigenis en herinnering: waar plaatsen we Primo Levi?

\section{Philippe Mesnard}

\section{(2) OpenEdition}

\section{Journals}

\section{Édition électronique}

URL : http://journals.openedition.org/temoigner/1425

DOI : $10.4000 /$ temoigner. 1425

ISSN : 2506-6390

Éditeur :

Éditions du Centre d'études et de documentation Mémoire d'Auschwitz, Éditions Kimé

Édition imprimée

Date de publication : 31 décembre 2014

Pagination : 77-90

ISBN : 978-2-84174-688-0

ISSN : 2031-4183

Référence électronique

Philippe Mesnard, «Entre témoignage et mémoire, quelle place pour Primo Levi ? 》, Témoigner. Entre histoire et mémoire [En ligne], 119 | 2014, mis en ligne le 01 juin 2015, consulté le 23 octobre 2020. URL : http://journals.openedition.org/temoigner/1425 ; DOI : https://doi.org/10.4000/temoigner.1425 


\section{Entre témoignage et mémoire, quelle place pour Primo Levi?}

$\rightarrow$ Par Philippe Mesnard

_ Registre

d'immatriculation du camp d'Auschwitz III. En cinquième ligne figure le nom de Primo Levi et son numéro 174517. a mémoire, telle que nous la concevons depuis quelque temps, a comme un défaut, un défaut de construction, si je puis dire, qui met à plat les profondeurs et les perspectives du passé comme si elle avait réponse à tout, en fonction de ses propres catégories et valeurs. Ainsi, un témoin aurait toujours été témoin, dès qu'il a commencé à écrire sur l'expérience pour laquelle il sera reconnu ; de même, il ne pourrait que contribuer à cette vaste et méritante entreprise qu'est la mémoire collective, sans imaginer qu'il puisse se lasser du devoir de mémoire, ni le critiquer. Pourtant, c'est ce qu’un regard attentif met en évidence à propos de Primo Levi, considéré comme un des témoins les plus « classiques » de son époque et au-dessus de toute ambivalence. Il suffit pour cela de s'intéresser aux conditions et aux circonstances d'écriture des différentes versions de Si c'est un homme et à la fatigue testimoniale qu'il éprouve à partir de la fin des années 1970 .

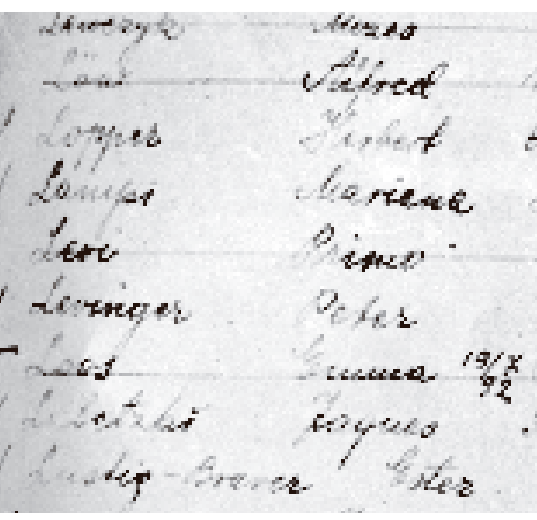

(1) Voir Claudio Pavone, Alle origini della Repubblica. Scritti su fascismo, antifascismo e continuità Turin, Bollati Boringhieri, 1995.

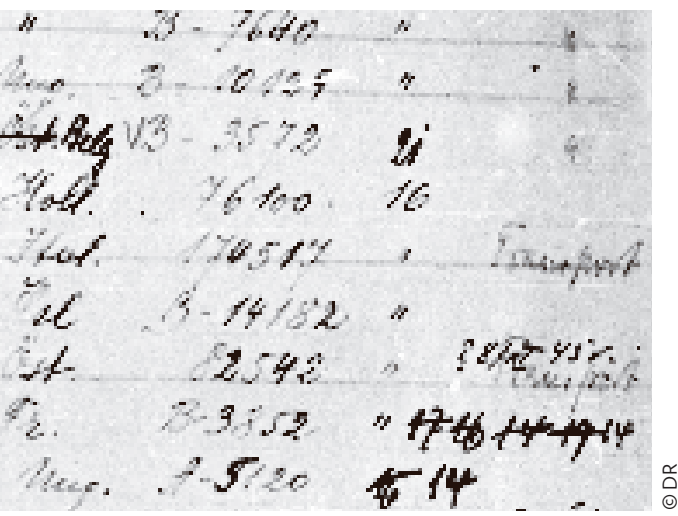

Mais il nous faut d'abord décrire rapidement le contexte et l'ambiance d'après-guerre dans lesquels Primo Levi vient à évoluer de retour d'Auschwitz.

\section{QUELLES COMMUNAUTÉS?}

Après vingt-quatre années de régime fasciste et de majorité consentante, l'après-guerre en Italie est le théâtre d'une survalorisation des figures antifascistes et de leurs martyrs tombés les « armes à la main ». Au milieu des ruines, les figures héroïques et salvatrices sont très attendues d'autant que le climat politique et social est extrêmement trouble. D’un côté, la présence fasciste est toujours perceptible, soit dans les administrations restées à l'abri de l'épuration ${ }^{1}$, soit comme groupe de pression politique, encore quasiment militarisé, démontrant que les racines de l'ancien régime n’ont pas été coupées. De son côté, le Parti communiste essaie de tirer profit de la situation pour instaurer 
Entre témoignage et mémoire, quelle place pour Primo Levi? (suite)

(1) Voir Claudio Pavone, Alle origini della Repubblica. Scritti su fascismo, antifascismo e continuità, Turin, Bollati Boringhieri, 1995.

(2) Paola Bertilotti, « La notion de déporté en Italie, de 1945 à nos jours. Droit, politiques de la mémoire et mémoires concurrentes », in Tal Bruttmann, Laurent Joly, Annette Wieviorka (dir.), Qu'est-ce qu'un déporté ? Histoire et mémoires des déportations de la Seconde Guerre mondiale, Paris, CNRS éditions, 2009, p. 377-402, ici p. 384.

(3) Guido Crainz, Autobiografia di una Repubblica. Le radici dell'Italia attuale, Rome, Donzelli editore, "saggine /150", 2009.

(4) Voir Keith Lowe, L'Europe barbare [2012], traduit de l'anglais par Johan Frederik Hel Guedj, Paris, Perrin, 2013.

(5) " seelische und emotionale Gemeinschaft ». Cf. Imre Kertész, "Holocaust als Kultur " [1992], in id., Eine Gedankenlänge Stille, während das Erschießzungskommando neu lädt, Hambourg, Rowohlt, 1999, p. $54-69$, ici p. 56 son monopole sur les régions. Face à quoi, la Démocratie chrétienne n’hésite pas à passer des alliances avec les fascistes d'après-guerre. Quant à la lourde question des persécutions antisémites, les instances gouvernementales tentent de la détourner en faisant porter son entière responsabilité sur les nazis ${ }^{2}$. Ainsi, le mythe d'une Italie fasciste, mais pas antisémite, et l'idée de l'Italien brava gente valorise l'image nationale au-delà des frontières alors qu'à l'intérieur, règne un climat de guerre civile larvée qui, avec son cortège de liquidations et d'épurations ${ }^{3}$, se prolonge jusqu'au début des années 1950, de même d'ailleurs que dans la plupart des pays d'Europe orientale ${ }^{4}$.

Quelle place les Juifs survivants, qui n’avaient pas ou peu été résistants, peuventils alors espérer? Quelle reconnaissance pour leur sort, humiliés et mis au ban de la société mussolinienne jusqu'en septembre 1943, puis, persécutés et déportés pour être assassinés ? Contrairement au lieu commun amplement promu et toujours véhiculé par quelques historiens, ils n’ont pas été systématiquement rejetés ni réduits au silence, mais accueillis, avec les « moyens du bord » alors que le navire national prenait encore l'eau, et le cap de sa trajectoire restait incertain.

Comme généralement, des associations deviennent les premiers véritables acteurs de mémoire. L'Associazione nazionale ex-deportati politici in Germania - exzebrati dei campi di eliminazione (ANED) est créée le 6 septembre 1945. Elle devient un véritable refuge pour les survivants et les familles des disparus. Des recherches sont entamées pour établir le nombre des déportés et leurs lieux de déportation. En 1947, dix-huit sections sont actives dans le pays. D’autres associations sont également actives, certaines se rallient à l'ANED, d'autres s'en éloignent pour des questions d'obédience politique car elle est, en effet, proche du Parti communiste. De même qu'en France ou en Belgique, ces associations d'ex-déportés forment ensemble un phénomène que l'on pourrait appeler une communauté mémorielle dont l'existence s'alimente de commémorations, d'expositions et d'importantes manifestations comme, en 1948, le transfert des cendres du déporté inconnu de Mauthausen au cimetière de Turin, et l'érection d'un monument au Déporté au cimetière principal de Milan. Primo Levi, de retour à Turin le 19 octobre 1945, fréquente immédiatement l'ANED; il se rend aux événements qui s'y organisent et entretient de chaleureuses relations avec ses membres dont les dirigeants ont tous été impliqués, la plupart à haut niveau, dans la résistance. En 1948, l'ANED est reconnue officiellement comme autorité morale. La communauté mémorielle, à travers ses associations les plus actives, lutte pour la reconnaissance des déportés et l'attribution d'un statut clairement défini. Elle est caractérisée par sa coloration politique au point que l'on peut dire que la construction mémorielle est un des aspects de la lutte politique qui se poursuit après-guerre.

Pas de tels enjeux pour la communauté testimoniale qui est formée par la convergence, souvent tacite ou, du moins, non concertée des témoignages sur une même histoire vécue et partagée. Renvoyant à une réalité informelle, l'expression « communauté testimoniale » reste très métaphorique, on pourrait l'associer à ce qu'Imre Kertész nomme une communauté d'êtres dépossédés de leur destin rappelant ainsi le titre de son ouvrage sur sa déportation, une communauté « spirituelle et émo- 
tionnelle ${ }^{5}$ », ou bien à la « communauté désœuvrée » de Maurice Blanchot. Celle-ci n'est pas seulement constituée des survivants, mais aussi des disparus dont on ne pouvait alors savoir ce qu'il en était advenu et des morts qui hantaient toujours l'univers mental de ceux qui avaient réchappé à la terreur nazie. Des liens se tissent, des réseaux non institués se constituent parallèlement aux associations pour savoir si un tel a survécu, si l'on a des nouvelles de celui-ci, si celle-là est bien morte à tel endroit. Cette communauté se tisse discrètement avec, pour seuls cadres et repères, les adresses des uns et des autres et leurs liens, parfois ténus, avec des associations. Si les deux communautés testimoniale et mémorielle se croisent, se rejoignent, elles ne se confondent pas.

De leur côté, bien que discrètes, des associations juives sont également actives. Le 26 septembre 1944, l'Union des Communautés juives italiennes crée un Comité de recherche des déportés juifs (CRDE). Si l'on est loin d'une claire distinction dont bénéficierait la spécificité de la Shoah, et loin de regarder en face les responsabilités accablantes du régime mussolinien dans la politique antisémite qui conduisit aux déportations génocidaires, il est en revanche facile de considérer que la terreur qui s'est abattue sur les antifascistes diffère de la curée aux Juifs qui a eu libre cours sous la République de Salò à partir de septembre 1943. Les deux persécutions ne répondaient pas aux mêmes objectifs ni fondements idéologiques. Mais à ce moment l'entreprise du CRDE se limite au recensement des absents et se rallie aux initiatives commémoratives des associations résistantes dans lesquels se retrouvent des victimes juives. Il tente également de gérer le flux de quantités de Juifs qui, chassés par les violences et l'instabilité de l'Est après-guerre, veulent gagner Israël. Primo Levi mentionne d'ailleurs certains de ceux-ci dans La Trêve dont il imaginera l'histoire à la fin de son roman Maintenant ou jamais (1981). Et s'il se trouve à appartenir à ces trois entités, à y participer, il n'en adopte pas moins, lorsqu'il commence à transcrire son expérience concentrationnaire, une position singulière que la qualité testimoniale ne permet pas d'identifier.

\section{QUELLES ÉCRITURES ?}

Durant la première année de son retour, Levi n’est pas encore un témoin au sens où on l'entend aujourd'hui. Le considérer comme tel serait même une reconstruction abusive interférant dans la compréhension du processus complexe qui a abouti à faire de lui un «témoin » - mais peut-il en être autrement pour quiconque revenu d'une telle expérience. Après quelques mois difficiles, il parvient aussi bien sur le plan professionnel qu'affectif à recouvrer un équilibre. Il trouve un travail de chimiste à Avigliana, non loin de Turin, et rencontre, au début 1946, Lucia Morpugo qu'il épouse un an plus tard. La singularité de son parcours de survivant ne tient pas tant à ce qu'il écrit - nombreux sont ceux qui passent alors par l'écriture qu'au fait que ce qu'il écrit entre dans un mouvement vaste et varié se constituant comme projet et expérience d'écritures en tant que tels. On peut ainsi affirmer que Levi est écrivain - sans appartenir à un champ littéraire duquel il se tiendra à dis-

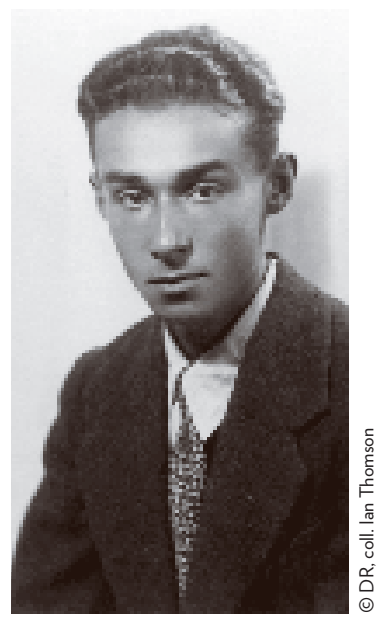

- Primo Levi étudiant, vers 1938. 
Entre témoignage et mémoire, quelle place pour Primo Levi? (suite)

(6) Par la suite, du théâtre, des romans, des essais, des chroniques journalistiques et intellectuelles viendront enrichir la diversité de son œuvre.

(7) Primo Levi, Conversations et entretiens 1963-1987, traduit de l'italien et de l'anglais par $\mathrm{T}$. Laget, Paris, Robert Laffont, 1998, p. 138

(8) Voir Primo Levi, Leonardo De Benedetti, « Rapport sur l'organisation hygiénico-sanitaire du camp de concentration de Monowitz pour Juifs », traduit de l'italien par Catherine Petitjean, in Primo Levi, Rapport sur Auschwitz, introduction et appareil critique Philippe Mesnard, Paris, Kimé, 2005. tance, tout en espérant sa reconnaissance - ; il est écrivain avant d'être témoin. Ce mouvement qui l'anime mobilise tous ses temps libres (pause déjeuner durant le travail et soirée incluses) pour, griffonnant des notes, transformer celles-ci en récit, ce mouvement, s'il ne peut être seulement identifié à une pulsion testimoniale, ne s'explique pas seulement comme reconstitution ou reconquête psychologiques. L'écriture, transcendante à ce souci de soi, ne peut y être circonscrite. Son extrême diversité, reflétant en partie la complexité de sa propre personnalité, exprime sa volonté de créer une scène intérieure où viendront se jouer et s'exprimer les échos de ses propres fantômes, tout en s'ouvrant au monde des lecteurs. Car l'écriture de Primo Levi restera toujours hautement transitive. Il va s'employer, durant son existence, à rédiger au même moment plusieurs textes aux écritures et aux genres radicalement différents. Si l'on ne s'en tient qu'aux premières années consécutives à son retour, il produit de la poésie, un texte documentaire, de la fiction proprement inventive sous forme de nouvelles et des formes brèves qui, comme des tableaux, retracent sa déportation et son expérience concentrationnaire ${ }^{6}$. L'ensemble, comme je vais tâcher de le montrer, rassemble les conditions de Si c'est un homme qui est encore, à ce moment, inclassable dans quelque genre que ce soit et qui ne porte même pas ce titre qui lui sera, pour ainsi dire, imposé au moment de la première publication du livre par son éditeur l'empruntant à un des poèmes rédigés, justement, à son retour de déportation.

Représentative de cette pluralité, la poésie constitue pour lui une expression primordiale qui demeurera toujours chargée d'inquiétudes et de douleur (celle des êtres perdus, celle de l'expérience des persécutions et du génocide, celle de l'expérience concentrationnaire - celles de la violence). «Les poésies viennent d'abord, je rentrais juste en Italie ${ }^{7} »$, confie-t-il plus tard. En effet, quinze poèmes suivent son retour jusqu'en 1946 avec une inflexion quand, au début de cette année, rencontrant Lucia Morpugo, il se sent revenir au bonheur de la vie et, ce faisant, être réapprovisionné en sens par l'amour. Ce qu'il rédige avec Leonardo De Benedetti est, en revanche, d'intention et de valeur précisément testimoniales. Les deux hommes publient dans le numéro de juillet-décembre 1946 de la revue Minerva Medica une version enrichie du rapport que l'Armée rouge leur avait commandé lorsqu'ils étaient au camp de Katowice en 1945, juste après être sortis d'Auschwitz et avant l'épopée du retour. Ce rapport adopte un style clair et concis dont l’intérêt littéraire dépasse sa valeur strictement documentaire ${ }^{8}$. Si De Benedetti y contribue par son diagnostic, Levi emporte le tout avec son style. On y trouve de véritables éléments matriciels de $S i$ c'est un homme. Une évolution significative marque ainsi le passage de la première version, expertise adressée à des militaires en quête de preuves, à la seconde destinée à un lectorat italien qui ne connaît rien de ce qui a eu lieu à l'autre extrémité de l'Europe. Cette première réécriture porte en elle la question de l'adresse testimoniale et, ce faisant, du projet à venir d'un survivant qui deviendra un des grands témoins des camps de concentration nazis et du génocide des Juifs.

Cet exercice, à la fois documentaire et testimonial, est synchrone à l'écriture de ce livre à venir, Si c'est un homme. D'ailleurs, un certain nombre de pages concernant 


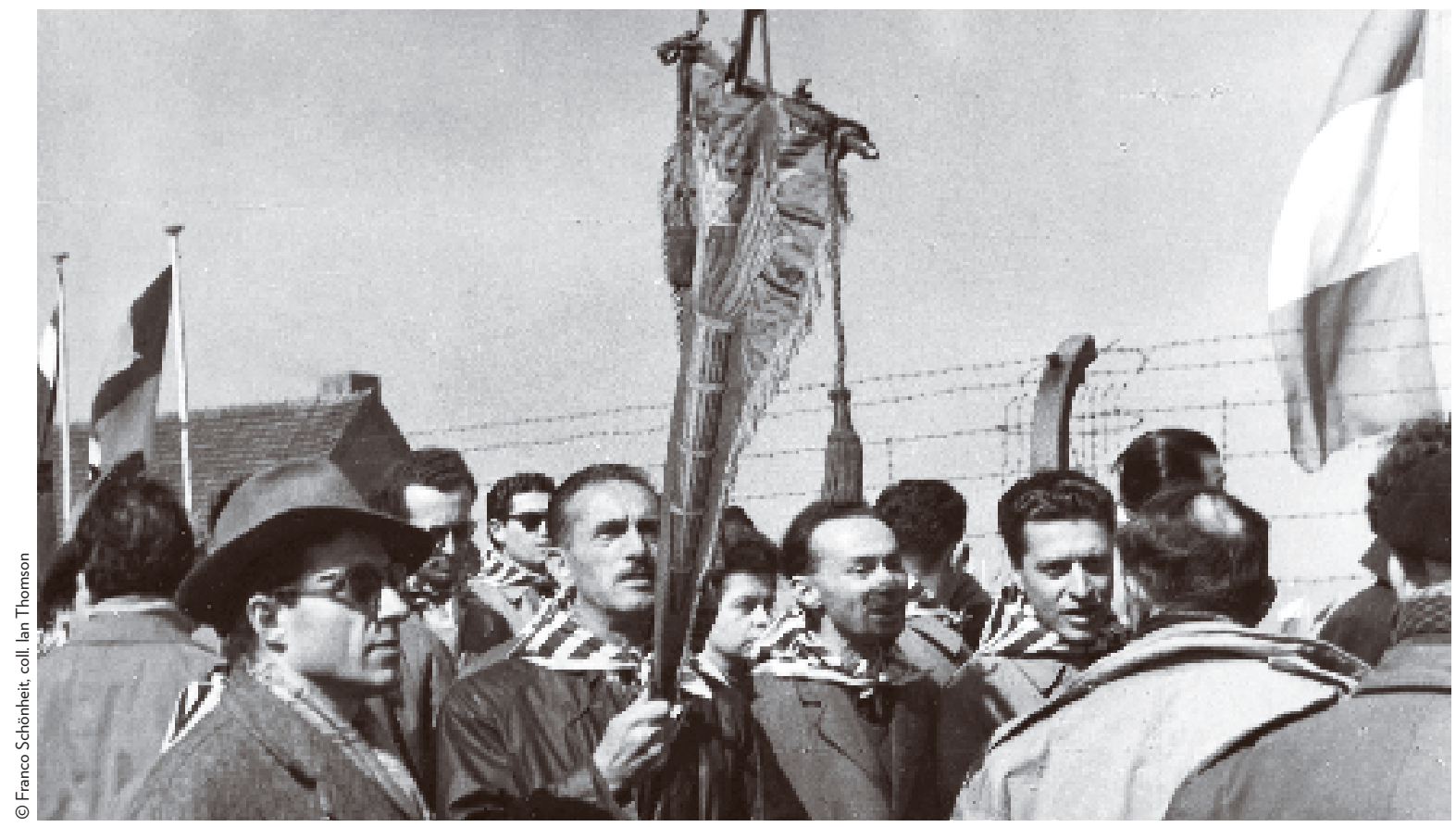

la fin de sa détention ne trouveront leur place que dans $L a$ Trêve, quinze ans après. C'est sur son lieu de travail, à Avigliana, que Levi consigne la plupart des épisodes de sa déportation et de son internement. Mais il le fait, pour ainsi dire, à reculons. Mi-février 1946, il entame d'abord la rédaction du dernier chapitre qui deviendra : « Histoire des dix jours », et c'est à la toute fin de décembre de la même année qu’il boucle ce qui ressemble alors plus à un recueil de tableaux ou d'épisodes quà un récit, et dont la succession chronologique n'est pas toujours une entrée pertinente pour en approcher la teneur et le sens.

Il est un chapitre sur lequel je voudrais m’arrêter car, peu commenté, sa genèse recèle pourtant une leçon fondamentale de témoignage selon Levi, à l'heure où cette expression et la transmission de son expérience n’avaient pas acquis, pour lui, toute son amplitude. Il s'agit du seizième chapitre, « Le dernier », qui se trouve être l'avant dernier du livre, et dont il écrit l'ébauche courant mars-avril 1946. Mais pour en saisir toute la dimension, il est important de le mettre en rapport avec une écriture que nous n'avons jusque-là pas commentée, celle, fictionnelle, des nouvelles dont Levi a rassemblé plusieurs volumes. Ici, il sera question de celle qui a pour titre le beau néologisme de « Mnémagogues »?

Mais qu'en est-il, tout d'abord, du « dernier » ? Le 7 octobre 1944 éclate, à Birkenau, la dernière révolte juive des camps d’anéantissement conduite par des Sonderkommandos. Elle a surtout un impact symbolique car elle ne bénéficie d'aucun

_ Primo Levi au mémorial du camp de Buchenwald, avril 1954.

(9) Primo Levi, « Les Mnémagogues » [1946], in id., Histoires naturelles [1966], traduit de l'italien par André Maugé, Paris, Gallimard, 1994, p. 11-21. 


\section{DOSSIER}

Entre témoignage et mémoire, quelle place pour Primo Levi? (suite)

(10) Primo Levi, Si c'est un homme [1958], Paris, Julliard / Pocket, 1987, p. 160 soutien de la part du très organisé réseau de résistance communiste qui, à l'instar des autres camps, prépare l'après-guerre et assure la survie de ses propres membres. Cette révolte a bénéficié de quelques complicités, entre autres, à l'intérieur du camp de Monowitz, dont la plupart a été identifiée par la Gestapo et exécutée. C'est cela que Primo Levi met en scène dans cet avant-dernier chapitre. La pendaison est un des moments récurrents des témoignages des camps nazis, il pouvait s'agir de condamnation à des tentatives d'évasion ou à des effractions à la loi SS comme, par exemple, l'homosexualité ou un trafic dont les SS n'auraient pas été bénéficiaires. C'est de l'exécution d'un résistant mêlé à ce projet de révolte qu'il s'agit ici. Le dispositif est théâtral : la potence avec le condamné et, face à lui, alignés sur l'Appelplatz, des milliers de déportés. Avant que la sentence ne soit appliquée, l'homme s'adresse à la foule assignée à une position obscène de spectateur : Kamaraden, Ich bin der Letzte (Camarades, je suis le dernier).

Je voudrais pouvoir dire que de notre masse abjecte une voix se leva, un murmure, un signe d'assentiment. Mais il ne s'est rien passé. Nous sommes restés debout, courbés et gris, tête baissée, et nous ne nous sommes découverts que lorsque l’Allemand nous en a donné l'ordre. La trappe s'est ouverte ${ }^{10}$.

C'est là un des rares passages de ce récit, comme des textes ultérieurs, où Levi aborde directement la question de la violence physique et de l'assassinat comme s'il

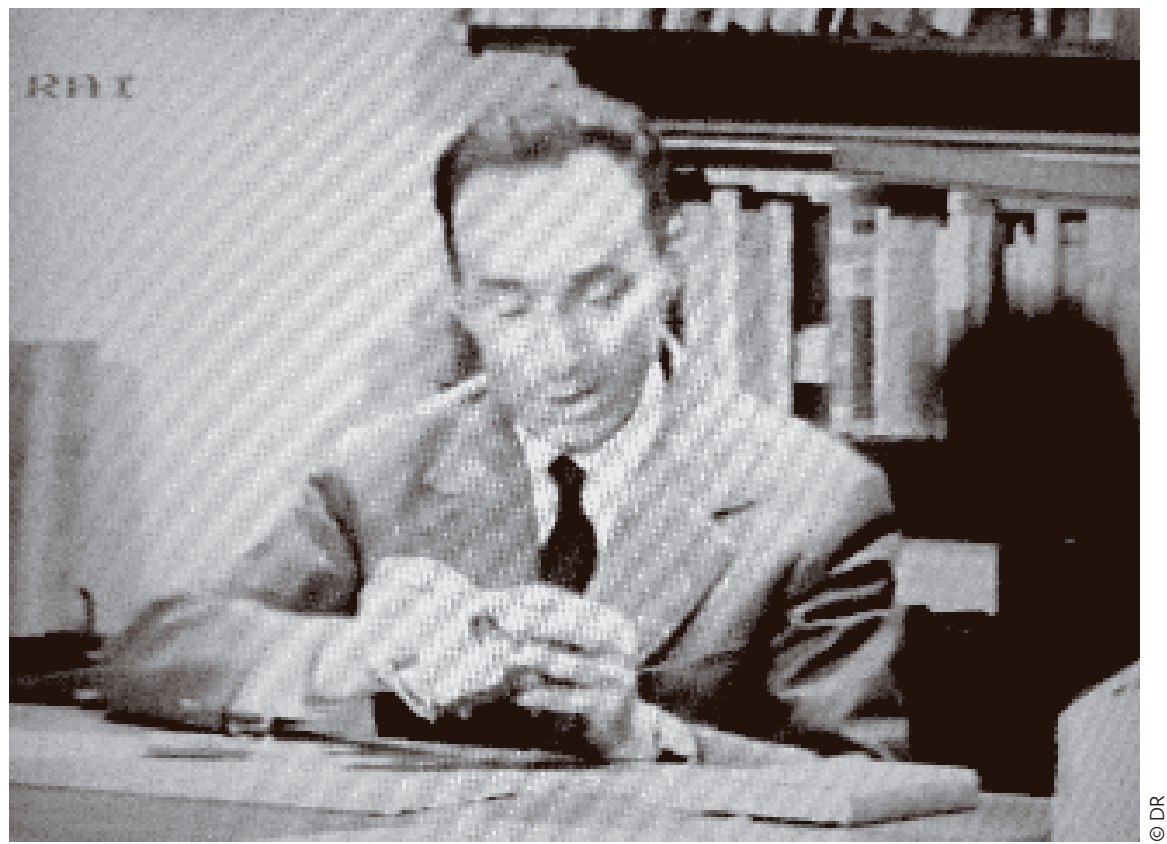

_ 27 septembre 1963.

Première émission télévisée de Primo Levi à la RAI. 
répugnait à devoir traiter la question de ce type de représentation. Le plus étonnant ne tient pas à cela, mais au fait que la scène est, dès 1946, entièrement reconstruite. Car ce sont en réalité trois détenus et non pas un, trois «prisonniers, accusés d'avoir tenté de planifier [promouvoir, littéralement] une insurrection simultanée [à celle des Sonderkommandos] au Lager de Monowitz ${ }^{11}{ }$. C'est à cette pendaison que Primo Levi assiste, en compagnie de De Benedetti, apprend-on dans une lettre datée du 17 octobre 1965 que rédige Giuliana Tedeschi et que cosignent Levi et De Benedetti.

Une scène avec les trois détenus ne satisfait pas à l'idée de transmettre des événements exemplaires, les faits trop fidèlement transcrits risqueraient d'interférer à la compréhension souhaitée par l'auteur. Dans un pays aussi catholique que l'Italie, l'image de la crucifixion dans ce contexte de souffrance serait trop facilement convoquée et trahirait l'événement réel. Levi reconstruit donc la scène pour mieux mettre en valeur, d'un côté, la résistance de laquelle dépend l'humanité dans l'homme, de l'autre, l'avilissement le plus abouti des déportés que le système serait, selon lui, parvenu à rejeter hors de l'humanité. Le seul appartenant encore à celle-ci est alors le pendu. Implacable constat que la plupart des commentaires éluderont.

Là, dès la rédaction de ces premières pages, réside une éthique du témoignage et de la communication exigeant d'être plus fidèle aux valeurs qu’à la réalité des faits. Cette éthique est confirmée par la façon dont Levi reconstitue également le célèbre passage, tellement commenté, du « Chant d’Ulysse » dont Jean Samuel confirme qu'ils ne l'ont pas vécu, Levi et lui, comme le récit le décrit ${ }^{12}$. Ainsi, non seulement Levi s'avère écrivain en devenant et pour devenir témoin, mais les éléments fondamentaux d'une éthique qui tâche de prendre la mesure de la violence extrême dont le sujet a été contemporain s'affirme avant de se faire reconnaître comme témoignage proprement dit.

Venons-en maintenant à cette nouvelle qui a pour titre : «Les Mnémagogues », répondant au genre spécifiquement littéraire de la science-fiction que l'on peut lire indépendamment de toute référence à l'univers concentrationnaire. «-Avez-vous jamais remarqué avec quelle force certaines odeurs évoquent certains souvenirs?», demande le savant, protagoniste du récit. L’odorat est un sens profondément marqué par l'expérience pour au moins deux catégories d'êtres auxquelles appartient Levi. Le chimiste, surtout quand celui-ci fabrique des peintures et des vernis comme il le fera jusqu’à la retraite, et le déporté qui est imprégné par l’odeur âcre des corps humains brûlant à jour continu dans les crématoires, et par celles du corps changé en vermine par l'insalubrité concentrationnaire. Agissant indépendamment de la volonté, l'odeur se révèle bien un terrible déclencheur de souvenir. Ce n'est donc pas un hasard si la première nouvelle qu'il achève tandis que, parallèlement, il poursuit la rédaction disparate des chapitres de Se questo è un uomo, soit consacrée à la mémoire des odeurs. Selon le savant des « Mnémagogues », se confiant à son interlocuteur :

[...] ily a des gens qui ne se soucient pas du passé, et laissent les morts ensevelir leurs morts. Il y en a d'autres, en revanche, qui se préoccupent du passé et que son évanouissement
(11) Voir fonds Primo Levi, Wiener Library, Londres, NB 257, boite 1406/2.

(12) Entretiens avec Jean Samuel en 2006. 
Entre témoignage et mémoire, quelle place pour Primo Levi? (suite)
(13) Primo Levi, « Les Mnémagogues », op. cit., p. 16.

(14) lbid.

(15) Ibid., p. 20. continuel attriste. Il y en a encore d'autres qui prennent soin de tenir un journal, jour après jour, afin que chaque chose les concernant soit sauvée de l'oubli, et qui conservent dans leur demeure et sur leur personne des souvenirs matérialisés : une dédicace sur un livre, une fleur séchée, une boucle de cheveux, des photographies et de vieilles lettres. Moi, de par ma nature, je ne puis penser qu’avec horreur à l'éventualité qu’un seul de mes souvenirs doive s'effacer, et j’ai adopté tous ces moyens, mais j’ai aussi inventé une nouvelle méthode ${ }^{13}$.

Ladite « méthode » est bien sûr ce qu’il appelle « des mnémagogues : des “suscitateurs" de souvenirs ${ }^{14} »$. Avec ce qui est évoqué dans la nouvelle : l'odeur siliceuse de pierres de montagne - Levi est un arpenteur chevronné des sommets alpins -, celle des salles scolaires poussiéreuses ou les sensibilités délicates de la «peau bien lavée, de poudre de riz et d'été ${ }^{15}$ ", l'heureux monde d'avant-guerre est ranimé, pourvu de cette douceur exagérément légendaire que le souvenir attache aux mondes d'avant le désastre. L'alchimie de la mémoire involontaire l'aide à trouver une distance qui ne peut pas lui venir de l'écriture même de son expérience charriant trop de morts et de corruption. Ainsi, dans le même temps qu'il transcrit et met en forme ce qu'il a vécu à Auschwitz, Levi recourt à la littérature pour trouver du sens par le détour de l'invention. Il s'échappe du camp pour y revenir avec plus de justesse. L'écriture de sa détention s'accomplit, quant à elle, suivant les règles de la mémoire volontaire : exercice rationnel, tout à fait en accord avec la démarche heuristique du chimiste, permettant au sujet de baliser les chemins de son propre passé au fur et à mesure qu'il les remonte - ce qui éclaire de façon évidente pourquoi c'est à rebours que Levi écrit Si c'est un homme. D’ailleurs, la spécificité volontaire de cette mémoire est déterminante dans l'élaboration de modèles et de scènes exemplaires comme celles des chapitres du « dernier » et du « chant d'Ulysse »; autrement dit, il y a un lien logique entre volonté et exemplarité dans le rapport que cette mémoire établit entre passé et transmission.

Mais cette mémoire, Levi le sait, ne représente qu'un pan de ce qui fait revenir le passé à soi. Si les mnémagogues sont étymologiquement des guides, c'est indépendamment de la volonté du sujet qu'ils agissent. De même que dans Wou le souvenir d'enfance, Perec dresse une scène fictionnelle pour y projeter ce qui, de son vécu, demeure enfoui dans des arcanes face auxquelles l'intentionnalité biographique reste dépourvue de moyens, de même, Levi déploie un espace science-fictionnel pour, dans le cadre de la science qui lui est familière, raconter le pouvoir immémorial de la mémoire involontaire capable de faire surgir des mondes entiers des limbes de l'oubli où ils demeuraient enfouis, ainsi que Proust l'a si remarquablement évoqué et, pour ainsi dire, enseigné.

Le récit de la réminiscence olfactive met en lumière combien certaines activités de la mémoire, fussent-elles menées par un ancien déporté, doivent être également distinguées de la mémoire, au sens social et collectif du terme, avec ses obligations et ses devoirs publics tels que Levi ne commencera à les assumer qu'une dizaine d'années plus tard. Quand il écrit cette première nouvelle d'une vaste série 
aboutissant ultérieurement dans deux recueils ${ }^{16}$, il ne pense pas à les publier, il les garde en réserve comme quantité d'autres projets. Écrire ces lignes l'aide à revenir parmi les vivants et, pour cela, participe à stabiliser les fantômes qui hantent celui qui a survécu (ce serait là comme la noble tâche de l'Orphée moderne qu'est le témoin, homme renaissant à lui-même, ce que Jean Cayrol nommait là l'écrivain « lazaréen ${ }^{17}$ ).

En ce sens, n'est-ce pas une façon de rater ce que le témoignage signifie et nous signifie que de le considérer en l'isolant du système d'écritures plurielles, voire d'expressions plurielles, et des tensions entre mémoires volontaire et involontaire dans lequel il se réalise et prend sens pour celui qui y recourt - et pour ceux qui le découvrent? C'est ce que révèlent les écritures de Primo Levi durant cette période de latence qui s’étend de la fin 1945 à 1947, jusqu’à la publication de la première version de Si c'est un homme. Dès lors que les textes entrent dans une phase de publication, qu'ils s'ouvrent au domaine public, leur sens se modifie. Qu’à ce moment, Si c'est un homme devienne un récit testimonial, cela est tout à fait possible, mais par inférence, alors que Primo Levi s'achemine vers cette position de témoin que nous lui connaissons, mais dans laquelle aussi il se sentira enfermé.

\section{RECONNAISSANCE ET RÉÉCRITURE TESTIMONIALES}

La façon dont s'organisent entre elles les différentes prépublications de Si c'est un homme, à travers des périodiques, du très communiste Amico del popolo de Vercelli, tiré à 10000 exemplaires, au très intellectuel Il Ponte de Florence, pour aboutir à la sortie du livre le 11 octobre 1947 chez De Silva, fait écho à notre mise en contexte liminaire : le Levi survivant et témoin public en devenir occupe une place dont celui qui écrit se tient éloigné, plus proche du Levi chimiste dont il partage le temps professionnel en s'y aménageant des pauses et des intervalles. C'est à l'occasion du dixième anniversaire de la fin de la guerre que le destin testimonial de Primo Levi se concrétise.

Dans l'Italie du début des années 1950, la réception des œuvres testimoniales ou romanesques liées aux persécutions nazies s'assouplit. Le Journal d’Anne Frank est publié en 1954. Les Armes de la nuit de Vercors et les deux livres de David Rousset paraissent également, de même pour Der Totenwald (Le bois des morts) dans lequel l'écrivain Ernst Wiechert, opposant au nazisme, raconte son internement à

(16) Histoires naturelles et Vice de forme sont réunis dans le même volume dans l'édition française.

(17) Jean Cayrol, « Pour un romanesque lazaréen » [1949], in id., Lazare parmi nous, Neuchâtel - Paris, La Baconnière - Le Seuil, 1950, p. 69-106.

- Couverture de la première édition de Si c'est un homme (1947). 
Entre témoignage et mémoire, quelle place pour Primo Levi? (suite)

(18) Primo Levi, L’Asymétrie et la vie [2002], traduit de l'italien par Nathalie Bauer, Paris, Robert Laffont, 2004, p. 21.

(19) Ibid.

(20) lbid.
Buchenwald en 1938. L'Espèce humaine de Robert Antelme, proche d'Elio Vittorini, prend place dans le catalogue Einaudi. Toujours en 1954, Si fa presto a dire fame (On a très vite criéfamine) de Piero Caleffi, déportéà Mauthausen, inaugure le renouveau des témoignages italiens dont les publications restent encore timides. L’Italie résistante, partisane, antifasciste, démocratique ou communiste se prépare à célébrer les dix ans de la victoire, ce que certaines associations voient précisément comme une opportunité pour faire reconnaitre nationalement leur importance historique et politique. Ce moment clé de l'Italie d'après-guerre, le sera également pour Levi qui se trouve en 1955 à prendre pour la première fois une position de témoin public et, la même année, à signer le contrat de la republication de Si c'est un homme par la prestigieuse Einaudi.

L’ANED, alors en perte de vitesse, choisit cette période propice pour lancer une vaste exposition photographique itinérante, la Mostra della Resistenza in Piemonte, qui, visant à faire connaître l'association du grand public et à étendre sa sphère d'influence, devient la clé de voûte d'une véritable politique de la mémoire ; Levi se saisit de son inauguration, à Turin, le 28 mai 1955, au Palazzo Madama, pour faire acte de présence et, par là même, s'engager ouvertement en commettant un texte retentissant «Déporté Anniversaire » (Deportati Anniversario) dans le numéro d'avril de Torino, la revue mensuelle de la ville distribuée gratuitement. Il y exprime ses craintes que la mémoire de la violence concentrationnaire et génocidaire ne se perde. « À dix ans de la libération des Lager, il est triste et significatif de devoir constater que, loin de se transformer en histoire, le sujet des camps d'extermination s'achemine, tout au moins en Italie, vers l'oubli le plus complet ${ }^{18}$. » Il insiste alors sur l'ampleur du massacre, le plus " gigantesque » de l'histoire et se focalise sur le fait qu'il a «pratiquement réduit à néant la population juive de plusieurs nations d'Europe orientale ${ }^{19} »$. Le passage suivant scelle la parole publique du militant de la mémoire qui vient de naître. À trop parler des Lager, dit-il,

[...] on risque d'être accusé, dans la meilleure des hypothèses, de vouloir se poser en victime, ou de manifester un amour gratuit pour le macabre; dans le pire des cas, de proférer des mensonges purs et simples, voire des outrages à la pudeur.

Ce silence est-il justifié ? Devons-nous le tolérer, nous autres rescapés ${ }^{20}$ ?

Nous autres rescapés, Levi, homme modeste, effacé, timide, discret - autant de qualificatifs dont ses proches usent pour le décrire - monte au créneau. Et il profère ce « Nous » qu'il avait déjà convoqué dans Si c'est un homme pour parler au nom des déportés, les morts et les vivants. Signant le 11 juillet 1955 le contrat pour la réédition du livre, sa réécriture va être amplement déterminée par cette nouvelle posture et par l'adresse testimoniale qui la sous-tend explicitement. Pendant deux ans, toujours avec d'autres écritures en parallèle, Levi remodèle aussi bien l'argumentation que le style de son tout premier livre et y incorpore ce dont il n'avait pas conscience quand il était juste revenu de ce lieu improbable, implanté par les nazis en Pologne, où l'on mourrait plus que l'on y vivait. 
Depuis dix ans, sa vision du système de la terreur concentrationnaire nazie s'est précisée et sa compréhension affinée. Plus sa connaissance progresse au rythme de ses lectures et de la parution d'ouvrages sur la question, plus il prend la mesure de l'ampleur du génocide des Juifs et de la systématicité de sa programmation. Son discours de témoin, dans l'acception publique du terme, est ainsi étayé par une connaissance dont son expérience ne pouvait pas l'avoir pourvu. L'assassinat systématique des enfants juifs ${ }^{21}$ lui apparaît dans sa brutalité sans précédent et, lui maintenant père de Lisa Lorenza et, bientôt, de Renzo, le convainc d'ajouter dans Si c'est un homme un certain nombre d'occurrences sur les enfants dans les camps comme celle de Schlome (Hurbinek n'apparaîtra finalement que dans La Trêve), la plus frappante de celles-ci restant l'évocation de la petite Emilia dans le convoi qui les a conduits, fin février 1944, de Fossoli à Auschwitz. Un paragraphe seulement d'une dizaine de lignes évoque la petite de 3 ans qui ne pouvait, suivant la loi des SS, survivre à la sélection. Cherchant le choc auprès du lecteur qui doit être littéralement saisi par l'horreur antisémite - il accumule les adjectifs en faveur d'Emilia d'une façon qui est, pour lui, tout à fait inhabituelle : «Curieuse, ambitieuse, gaie, intelligente. » La volonté de toucher le lecteur ne se limite pas à cet ajout, mais se transmet à travers l'ensemble des modifications apportées à la seconde version. Il en est ainsi des nouvelles mentions et citations de L'Enfer de Dante qui sont autant de passerelles que Levi établit entre le lecteur italien familier de La Divine comédie et son témoignage, pour mettre celui-ci plus facilement à portée de son public.

Mais l'ouverture vers le lectorat de ce récit en passe de devenir canonique n'est certainement pas aussi flagrante que dans les deux passages suivants. D'abord, dans la version de 1947, Levi commence directement par son internement au camp de transit de Fossoli, l'épisode de son arrestation et la question même de ses rapports avec le maquis relève d'une autre histoire - pourquoi en parlerait-il ? Dans celle de 1958, il ajoute deux pages sur ce sujet parce que c'est établir un lien, non pas avec son histoire d'avant, mais avec l'actualité de la mémoire qui, dans les années 1950, est portée par les associations de résistants, non de la communauté hébraïque, dans lesquelles se retrouvent la plupart des Juifs italiens revenus des camps nazis. Ensuite, il introduit à la suite du deuxième chapitre celui que l'on connaît sous le titre d' « Initiation » - absent donc du texte de 1947 - où il met en scène un ancien officier austro-hongrois dénommé Steinlauf, directement sorti de La Marche de Radetzki de Joseph Roth, avec lequel il entame un dialogue sur la nécessité de s'adapter aux conditions du camp pour y survivre, finissant par retourner l'argumentation de son interlocuteur qui, aux yeux du public piémontais, reste emblématique de la rigidité de ceux qui ont historiquement dominé le pays jusqu'au Risorgimento. Cette reconstruction est encore un appel à méditer sur la vie en condition extrême sous un régime de terreur alors que, quinze ans après la fin de la guerre, l'Italie entre dans une période de prospérité.

Je ne m'attarderai pas sur la réception de cette nouvelle édition qui doit, en fait, attendre 1963, le succès de librairie de La Trêve retraçant son retour de Pologne à Turin, pour que soit reconnue la force de sa composition et de son écriture. Dans
(21) Voir Catherine Coquio et Aurélia Kalisky (dir.), L'Enfant et le génocide. Témoignages sur l'enfance pendant la Shoah, Paris, Robert Laffont, 2007. 


\section{DOSSIER}

Entre témoignage et mémoire, quelle place pour Primo Levi? (suite)

_ Adaptation théâtrale de Si c'est un homme, jouée pour la première fois le 18 novembre 1966 au théâtre Carignano de Turin. les années 1960 et 1970, Primo Levi est alors le témoin que l'on imagine. Il se rend dans les écoles, il est sollicité à la télévision, il écrit dans La Stampa, dans le Corriere della Sera... ses chroniques dans des quotidiens et des magazines constituent désormais une activité régulière. Il a sa place dans la grande presse. Il polémique, par exemple, avec Manganelli, il se retrouve dans des débats avec Paolo Volponi ${ }^{22}$ ou bien à l'occasion de la sortie du contesté Clé à molette qui s'attire les critiques de «classe» de l'extrême-gauche. Il intervient publiquement contre les Brigades rouges, dénonce l'assassinat d'Aldo Moro, la vague de films porno-nazis, le négationnisme et prend position, en 1985, à propos l'Historikerstreit. Il enregistre de nombreuses émissions de télévision. Il participe à la conception du monument du pavillon italien d'Auschwitz dont le projet, voyant le jour en 1972, n'aboutit que dix ans plus tard. Son texte devient le texte officiel de la déportation italienne à Auschwitz; il rédige aussi celui de la plaque commémorant la déportation dans la gare de Turino Porta Nuova. Parallèlement à cela, il poursuit l'écriture de son œuvre et, à travers elle, sa réélaboration de Si c'est un homme, aboutissant à une adaptation théâtrale qui est représentée à Turin en 1966, à des versions scolaires, mais aussi, finalement, à un ensemble d'essais pour lesquels la critique de la et de sa position de témoin devient la clé de voûte. Je voudrais donc conclure sur cette question qui jette une sombre lumière sur sa relecture, quarante ans après, de son expérience testimoniale, et sur la réception durant les années 1980 de la parole des témoins (il se suicide le 11 avril 1987).

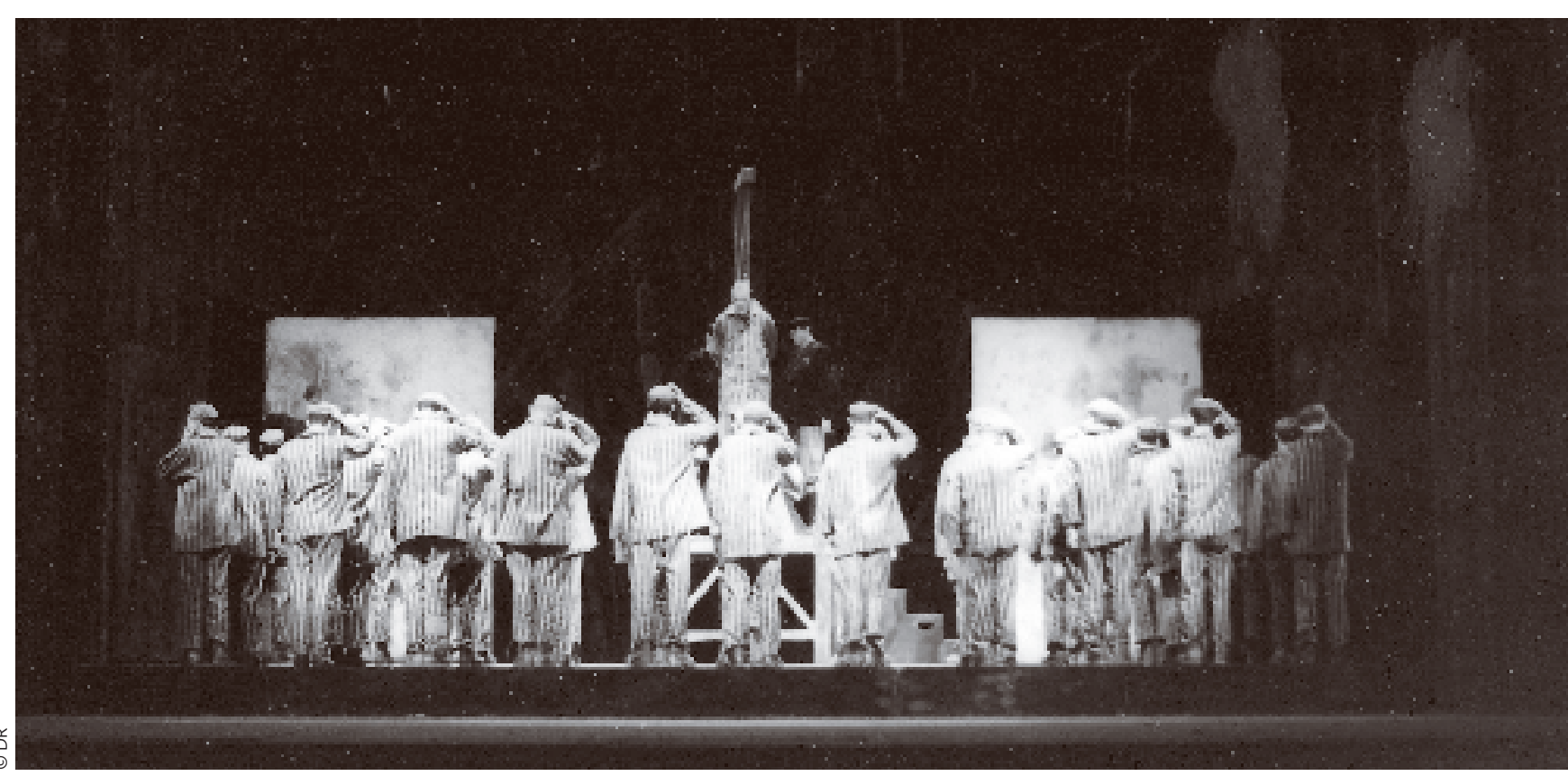




\section{FATIGUE TESTIMONIALE ET CRITIQUE}

Conjonction des années 1970 et de sa dépression, la décade suivante est placée, pour Levi, sous le signe de l'écroulement de la fonction testimoniale.

Ainsi, je suis devenu un « rescapé professionnel », presque un mercenaire... (rires) Mon expérience de l'époque est profondément altérée. Elle est altérée par une foule de réflexions postérieures, de conversations, comme celle quej’ai avec vous en ce moment. J’ai accordé un très grand nombre d'interviews, et tout cela s'interpose entre l'expérience authentique et aujourd'hui [...]. Tout cela dresse une grande barrière ${ }^{23}$.

Cohabiter avec le souvenir revient, pour lui, à une épreuve qui se tourne en un profond mal-être. Que le survivant (celui qui est sorti du camp) tire le témoin (celui qui a vécu dans le camp) vers la lumière de l'existence, cela génère chez Levi un profond dilemme : tout en racontant son expérience, le survivant s'en éloigne. Les mots ne l'assistent plus comme avant, Levi s'en rend compte désormais chaque fois qu'il se trouve en situation de témoigner. Ils ne servent plus de médiation pour maintenir ensemble le témoin avec le survivant. Ils ont perdu ce pouvoir d'exprimer la réalité qu'ils désignent (réalité vécue, de l'expérience, de ce qui a eu lieu).

Je ne vais plus volontiers dans les écoles. D’un côté, je l'avoue, je suis las de m’entendre toujours poser les mêmes questions. D'un autre côté, j'ai l'impression que mon langage est devenu insuffisant, que je parle une langue différente. Et puis, je dois avouer que j’ai été touché au vif par une des dernières expériences que j’ai faites dans une école, où deux enfants, deux frères, m’ont lancé d'un ton sans réplique : «Pourquoi venez-vous encore nous raconter votre histoire, quarante ans après, après le Viêt Nam, après les camps de Staline, la Corée, après tout cela... pourquoi ?» Et je dois dire que je suis resté bouche bée, sans voix, poussé dans mes retranchements, dans ma condition de rescapé à tout prix ${ }^{24}$.

Son amie Edith Bruck mène une expérience similaire. Après avoir écrit une dizaine de livres, des récits autobiographiques et testimoniaux, de la poésie, nourris aux thèmes de l'exil, de la solitude, de la judéité et méditant sa propre expérience, elle publie Signora Auschwitz reprenant l'expression par laquelle une étudiante la considère.

D’emblée, Levi abat ses cartes les plus fortes : les témoins ont-ils correctement rempli leur tâche? La réception du côté des jeunes n'est-elle pas faussée? Il s'adresse alors à la communauté des rescapés en convoquant ce nous si souvent présent dans Si c'est un homme. "Avons-nous été capables, nous qui sommes rentrés, de comprendre et faire comprendre nos expériences ${ }^{25}$ ? » L'attaque n'est pas anodine et couvre les deux premières pages de l'essai intitulé « La zone grise » dans le recueil Les Naufragés et les rescapés, qu’il publie en 1985. « Nous avons tendance à simplifier aussi l'histoire », par trois fois, il répète « simplifier », « simplification ». On n’a pas encore pris la mesure critique de ce constat dont Levi nous tend le miroir quand,
(22) Primo Levi entre en polémique avec l'écrivain Giorgio Manganelli qui attaque dans le Corriere della Sera daté du 3 janvier 1977 son article « De l'écriture obscure », paru dans $L a$ Stampa quelques jours avant, et dans lequel Primo Levi critique notamment Paul Celan et Georg Trakl pour l'herméticité de leur poésie, faisant valoir avec une certaine intransigeance un principe de communicabilité que ne partage bien évidemment pas l'auteur de La letteratura come menzogna (1967). Avec Paolo Volponi, il s'agit plus anecdotiquement d'une tribune qui a lieu le 9 septembre 1979, au moment de la fête de L'Unità, équivalent de la fête de l'Humanité pour les Français, lors de laquelle Primo Levi conteste l'esthétique obscurantiste de l'écrivain reconnu de gauche et sa posture romantique anticapitaliste.

(23) Primo Levi, Conversations et entretiens, op. cit., p. 252.

(24) Primo Levi, Le devoir de mémoire [1983], entretien avec Anna Bravo et Frederico Cereja, Paris, Mille et une nuits, 1994, p. 37.

(25) Primo Levi, « La zone grise ", Les Naufragés et les rescapés (1985), traduit de l'italien par André Maugé, Paris, Gallimard, 1987, p. 36. 
Entre témoignage et mémoire, quelle place pour Primo Levi? (suite)
(26) Ibid., p. 37.

(27) lbid.

(28) Primo Levi, Si c'est un homme, op. cit., p. 7. précisément au milieu des années 1980, sont lancés les grands projets d'enregistrement de témoignage.

Les témoins de l'époque ne l'entendent pas et, d'une certaine manière, que Levi soit délégué par nombre d'entre eux à parler à leur place, ne facilite pas cette prise de conscience de leur part. Levi souligne que l'on ne peut comprendre une telle complexité en termes d'ami et d'ennemi.

C'est certainement la raison de l'énorme popularité des sports spectaculaires, tels que le football, le base-ball et la boxe, où les concurrents sont deux équipes ou deux individus, bien distincts et bien identifiables, et où à la fin de la partie il y aura des vainqueurs et des vaincus ${ }^{26}$.

À ce propos, il interpelle les jeunes qui, à ses yeux, semblent tellement avoir besoin « de séparer le mal du bien, de pouvoir prendre parti », qu'ils veulent qu’il y ait «ici les justes, là les réprouvés ». Eux qui « demandent que les choses soient claires, que la séparation soit franche ». Les stéréotypes caractérisent aussi la mentalité de ce public envers lequel il éprouve maintenant un sentiment de grande déception : « leur expérience du monde étant pauvre, ils n'aiment pas l'ambiguïté27. »

Levi sait que les témoignages des déportés ont, en convoquant des modèles et des stéréotypes, gommé la zone grise et, ce faisant, interféré à faire comprendre l'univers concentrationnaire en le simplifiant. C'est le paradoxe d'une transmission qui, pour rendre son objet accessible, le dénature, lui ôtant une partie de sa complexité. Luimême a beaucoup moralisé son témoignage et a commencé de témoigner bien avant d'avoir exploré la zone grise. Ses mots ne l'épargnent pas et certainement viennent-ils même le heurter en premier, lui qui a tenté de délivrer une vision « dépassionnée $e^{28}$ » et qui se retrouve à faire de la zone grise une notion de combat contre les témoins?

$$
\text { 怾 }
$$

Les réécritures de Si c'est un homme jusqu’à son dernier essai Les Naufragés et les rescapés retracent l'exigence d'un homme qui ne s'est jamais satisfait de sa propre position, ni du confort qu'elle aurait pu lui apporter, avec la reconnaissance et les honneurs dévolus à un grand écrivain doublé d'un témoin majeur dont le nom circulait parmi ceux des nobélisables. Or, Primo Levi n’a pas hésité à détricoter ses propres légendes pour répondre à l'exigence impérieuse de penser, penser non seulement l'expérience d'un système de terreur qui avait pour finalité l'abolition de la pensée et de la culture avant la destruction de l'humain, mais aussi la condition instable et sans apaisement de celui qui se retrouve entre témoignage et mémoire. I 